AIAA-92-2261-CP

\title{
A NEW FLEXIBLE BODY DYNAMIC FORMULATION FOR BEAM STRUCTURES UNDERGOING LARGE OVERALL MOTION
}

\author{
W. J. Haering \\ Senior Project Engineer \\ General Motors Corporation \\ Warren, Michigan \\ R. R. Ryan* \\ Executive Vice President and Chief Operating Officer \\ Mechanical Dynamics Incorporated \\ Ann Arbor, Michigan \\ R. A. Scott \\ Professor, Department of Mechanical Engineering and Applied Mechanics \\ The University Of Michigan \\ Ann Arbor, Michigan
}

\begin{abstract}
A new flexible body dynamic formulation, called the Augmented Imbedded Geometric Constraint (AIGC) approach, for beam structures undergoing large overall motion is developed. It is restricted to small elastic deformations of the beam about the large overall motion. The formulation outlined herein pertains to two-dimensional motion and deformation of a single beam when the overall motion is prescribed as a function of time. The formulation can be easily extended to beam assemblies undergoing arbitrary motion in three-dimensional space. Elastic deformation is characterized by the superposition of a number of assumed global shape functions developed from a substructuring method. The motion of the system is governed by a set of differential and algebraic equations. The algebraic constraints arise from enforcement of the boundary conditions. The AIGC approach improves upon two existing approaches by allowing the solution of two disparate classes of elasto-dynamics problems with a single formulation, demonstrated by simulations for several verification problems. The problems are ones in which the lateral deformation of the beam is dominated by either bending or membrane behavior. Because the new formulation is problem independent, it is applicable to beam problems where the dominant stiffness effects are not known beforehand.
\end{abstract}

\section{Introduction}

The study of the coupling between overall dynamic motion and local deformation of structures has become important with the advent of the space-age, since the interaction is more pronounced with the relatively flexible structures common in spacecraft design. The effects of such coupling are important in the aeronautics industry and can be seen, for example, in helicopter blade response. High speed motion of robotic arms and rapid ground transportation systems are other areas in which the coupling effects are imnortant.

\footnotetext{
* Member AIAA
}

One approach to studying flexible body dynamics is through the use of finite element methods ( see for example, Simo and $\mathrm{Vu} \mathrm{Quoc}^{1,2}$ and Christensen and Lee ${ }^{3}$ ). Another strategy is to use rigid body dynamic approaches which have been modified to include the flexibility effects. Kane, Ryan and Bannerjee ${ }^{4}$ used this strategy to study beams undergoing large overall motion of a prescribed nature.

The technique introduced in reference [4] was restricted to systems with known overall motion. Ryan 5 extended that formulation to allow solutions when forces/torques are applied. Subsequently, Yoo ${ }^{6}$ has shown that the approach in references [4-5], which he refers to as the Imbedded Geometric Constraint (IGC) approach, fails to produce the correct result for problems where the lateral deformation of the beam is dominated by membrane stiffness. Yoo demonstrated that his formalism, which he refers to as the Nonlinear Strain Displacement (NSD) approach, handles such problems quite successfully. However the NSD method does not reliably solve problems in which lateral deformations are dominated by bending stiffness, which are handled very well by the IGC approach.

A new approach called the Augmented Imbedded Geometric Constraint (AIGC) approach, is presented herein. It allows the solution of problems where the lateral deflection of the beam is dominated by either bending or membrane stiffness. This formulation is a modification of the IGC approach. It is problem independent and, therefore, is applicable to structural dynamics problems where the dominant effects are not known before hand.

Only small local deformations of the beam are considered. An Euler-Bernoulli model of the beam transverse flexibility, assuming linear elastic, isotropic behavior, is used. A set of ordinary differential equations (ODEs) describing the flexible body dynamic behavior of the beam is developed using Kane's ${ }^{7}$ method. That portion of the development of the AIGC approach is identical to that for the IGC approach. Differential algebraic equations (DAEs) of motion for the AIGC approach are generated by developing a set of algebraic constraints enforcing the physical boundary conditions for the beam. The spatial representation of the deformation is achieved through the use of global shape functions which are based on the substructuring techniques of Craig and Bampton ${ }^{8}$. 
The plan of the paper is as follows. In the next section the system differential equations are derived. Then a general method for enforcing boundary conditions is described and implemented, resulting in a set of differential algebraic equations of motion. Numerical results for two benchmark problems, generated using several formulations, are then presented and compared.

\section{Development of System Differentia_Equations}

The model for a two-dimensional beam undergoing large overall motion and small local deformation is shown in Figure 1 . This model consists of a rigid body $A$ and a flexible beam $B$ of length $L$. A dextral set of mutually perpendicular unit vectors, $\vec{a}_{1}, \vec{a}_{2}, \vec{a}_{3}$, are fixed in A and directed as shown in Figure 1 . The centroidal axis of the beam is assumed to be coincident with the elastic axis, and is parallel to the $\vec{a}_{1}$ direction when the beam is undeformed. Point $P_{0}$ located a distance $\mathrm{x}$ along the undeformed centroidal axis represents a generic point on the beam. After deformation, that point lies at a new position which is labeled point $P$. The position vector from point $O$ to point $P$ is given by:

$\vec{p}^{\overline{O P}}=\left(x+u_{1}\right) \vec{a}_{1}+u_{2} \vec{a}_{2}$

where $u_{1}$ and $u_{2}$ are the $\vec{a}_{1}$ and $\vec{a}_{2}$ measures of the beam deformation.

An additional variable of interest is $\mathrm{s}$, the stretch of the centroidal axis of the beam. After deformation, point $P$ is located at a distance $\mathrm{x}+\mathrm{s}$ measured along the deformed centroidal axis.

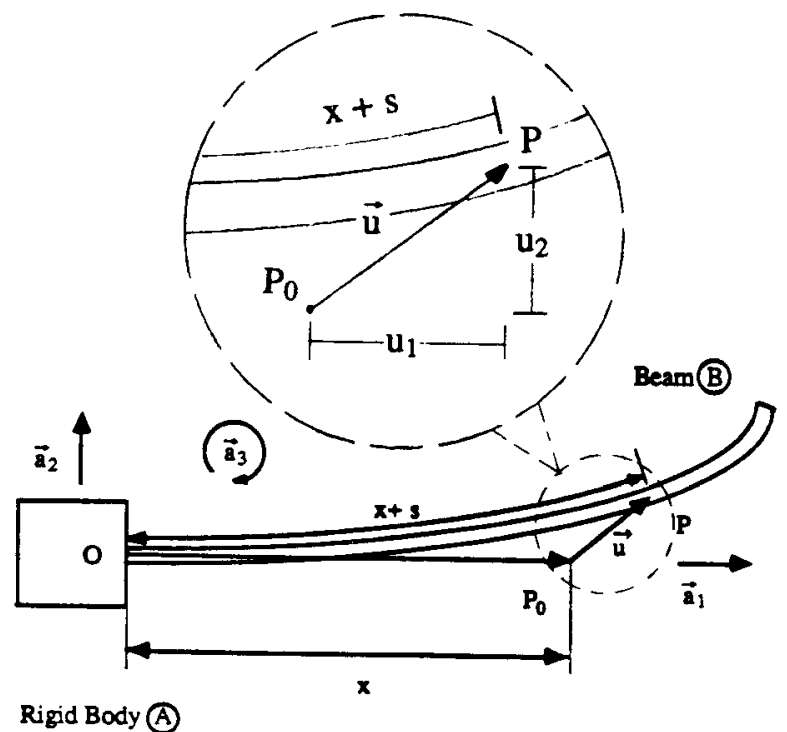

Figure 1 -- Beam Description and Deformation Measures

The equations of motion are derived using Kane's dynamical equations:

$\mathrm{F}_{\mathrm{r}}+\mathrm{F}_{\mathrm{r}}^{*}=0, \quad \mathrm{r}=1,2, \ldots, \mathrm{p}$ where $p$ is the number of system degrees of freedom, and $F_{r}$ and $F_{I}{ }^{*}$ are the $r$ th generalized active force and generalized inertia force, respectively.

The generalized inertia force, $\mathrm{F}_{\mathrm{r}}{ }^{*}$, is associated with the mass distributed along the length of the beam and is given by:

$F_{r}^{*}=-\int_{0}^{L} \rho \stackrel{N_{V_{r}}}{P} . N_{a} P d x, r=1,2, \ldots, p$

where $\rho$ represents the beam mass per unit length and ${ }^{N} \rightarrow P$ represents the acceleration of point $P$ in the Newtonian reference frame $N$. ${ }^{N} \vec{v}_{r} P$ is the $t$ th partial velocity of ${ }^{N} \vec{v}^{P}$, the velocity of point $P$ in the Newtonian reference frame $N$, and is a fundamental element in the formulation of Kane's dynamical equations. The partial velocity is defined as

$\mathrm{N}_{\mathbf{r}} \mathrm{P} \equiv \frac{\partial^{\mathrm{N}} \overrightarrow{\mathrm{v}}^{\mathrm{P}}}{\partial \mathrm{u}_{\mathrm{r}}^{*}} \quad, \quad \mathrm{r}=1,2, \ldots, \mathrm{p}$

where $u_{\mathrm{r}}^{*}$ are the generalized speeds. In this development they are chosen as

$\mathrm{u}_{\mathrm{r}}^{*}=\dot{\mathrm{q}}_{\mathrm{r}}, \quad \mathrm{r}=1,2, \ldots, \mathrm{p}$

where $\mathrm{q}_{\mathrm{r}}$ is the $\mathrm{r}$ th generalized coordinate (specified later), and a dot denotes a time derivative.

The generalized active force, $F_{\mathrm{r}}$, derived from a potential function describing the strain energy, $\mathrm{V}$, is

$F_{r}=-\frac{\partial V}{\partial q_{r}}, r=1,2, \ldots, p$

Detailed expressions for the generalized inertia and generalized active forces will now be developed.

\section{Generalized_Inertia_Force}

As seen in equation 3 , the acceleration and velocity of point $P$ in a Newtonian reference frame are needed to form the generalized inertia force. To facilitate their formulation, the planar motion of the rigid base, relative to the Newtonian reference frame, can be described by its angular velocity, ${ }^{N} \vec{\omega}^{A}$, and the translational velocity of some point $\mathrm{O}, \mathrm{N}_{\mathrm{v}}^{\mathrm{O}}$, fixed on the base.

${ }^{N} \vec{\omega}^{A}=\omega_{3} \vec{a}_{3} \quad, \quad N \vec{v}^{O}=v_{1} \vec{a}_{1}+v_{2} \vec{a}_{2}$

where $\omega_{3}$ is the $\vec{a}_{3}$ measure of the angular velocity of body $A$, and $v_{1}$ and $v_{2}$ are the $\vec{a}_{1}$ and $\vec{a}_{2}$ measures of the translational velocity of point $O$.

The velocity of point $P$ in the Newtonian reference frame, ${ }^{N} \vec{v} P$, is

$\mathbf{N} \vec{v}^{P}=\left[v_{1}+\dot{u}_{1}-\omega_{3} u_{2}\right] \vec{a}_{1}+\left[v_{2}+\dot{u}_{2}+\omega_{3}\left(x+u_{1}\right)\right] \vec{a}_{2}$

The Newtonian acceleration of point $P,{ }^{N} \vec{a}$, is

$N \vec{a}^{P}=\left[\dot{v}_{1}+\ddot{u}_{1}-\dot{\omega}_{3} u_{2}-2 \omega_{3} \dot{u}_{2}-\omega_{3} v_{2}-\omega_{3}^{2}\left(x+u_{1}\right)\right] \vec{a}_{1}$ 
$+\left[\dot{v}_{2}+\ddot{u}_{2}+\dot{\omega}_{3}\left(x+u_{1}\right)+2 \omega_{3} \dot{u}_{1}+\omega_{3} v_{1}-\omega_{3}^{2} u_{2}\right] \vec{a}_{2}$

In the current (as well as the IGC) approach, the variables s and $\mathrm{u}_{2}$ are used to describe the deformation. They are represented as follows:

$s=\sum_{j=1}^{v} \phi_{1 j}(x) q_{j}(t) \quad, \quad u_{2}=\sum_{j=1}^{v} \phi_{2 j}(x) q_{j}(t)$

where $\phi_{1 \mathrm{j}}$ and $\phi_{2 \mathrm{j}}$ are shape functions, $\mathrm{q}_{\mathrm{j}}$ are the generalized coordinates, and $v$ is the total number of functions used to describe the beam deformation. The specific choice of the shape functions, $\phi_{1 \mathrm{j}}$ and $\phi_{2 \mathrm{j}}$ will be presented later.

Since $s$ and not $\mathrm{u}_{1}$ is chosen as a deformation measure, the following geometric relationship, given in reference [6], between the variables $s, u_{1}$, and $u_{2}$ will be used to develop expressions for $u_{1}, \dot{u}_{1}$, and $\ddot{u}_{1}$ which appear in equations 9 and 10

$x+s=\int_{0}^{x}\left[\left(1+\frac{\partial u_{1}}{\partial \sigma}\right)^{2}+\left(\frac{\partial u_{2}}{\partial \sigma}\right)^{2}\right]^{\frac{1}{2}} d \sigma$

where $\sigma$ is a dummy variable of integration. After performing a binomial expansion on the right hand side of equation 13 and neglecting terms higher than degree two, the following expression can be obtained

$$
\mathrm{u}_{1}=\mathrm{s}-\frac{1}{2} \int_{0}^{\mathrm{x}}\left(\frac{\partial \mathrm{u}_{2}}{\partial \sigma}\right)^{2} \mathrm{~d} \sigma
$$

Substituting the relationships for $\mathrm{s}$ and $\mathrm{u}_{2}$ from equations 11 and 12 into equation 14 yields

$u_{1}=\sum_{j=1}^{v} \phi_{1 j} q_{j}-\frac{1}{2} \int_{0}^{x} \sum_{j=1}^{v} \sum_{k=1}^{v} \phi_{2 j}^{\prime} \phi_{2 k} q_{j} q_{k} d \sigma$

where a prime denotes a partial derivative with respect to $x$. From equations 12 and 15 , it follows that

$$
\begin{gathered}
\dot{\mathrm{u}}_{1}=\sum_{\mathrm{j}=1}^{v} \phi_{1 \mathrm{j}} \dot{\mathrm{q}}_{\mathrm{j}}-\int_{0}^{\mathrm{x}} \sum_{\mathrm{j}=1}^{v} \sum_{\mathrm{k}=1}^{v} \dot{\phi}_{2 \mathrm{j}}^{\prime} \phi_{2 \mathrm{k}}^{\prime} \mathrm{q}_{\mathrm{j}} \dot{\mathrm{q}}_{\mathrm{k}} \mathrm{d} \sigma \\
\ddot{\mathrm{u}}_{1}=\sum_{\mathrm{j}=1}^{v} \phi_{1 \mathrm{j}} \ddot{\mathrm{q}}_{\mathrm{j}}-\int_{0}^{\mathrm{x}} \sum_{\mathrm{j}=1}^{v} \sum_{\mathrm{k}=1}^{v} \dot{\phi}_{2 \mathrm{j}} \phi_{2 \mathrm{k}}^{\prime} \dot{\mathrm{q}}_{\mathrm{j}} \dot{\mathrm{q}}_{\mathrm{k}} \mathrm{d} \sigma \\
\int_{0}^{\mathrm{x}} \sum_{\mathrm{j}=1}^{v} \sum_{\mathrm{k}=1}^{v} \phi_{2 \mathrm{j}}^{\prime} \phi_{2 \mathrm{k}}^{\prime} \mathrm{q}_{\mathrm{j}} \ddot{\mathrm{q}}_{\mathrm{k}} \mathrm{d} \sigma
\end{gathered}
$$

$$
\dot{\mathrm{u}}_{2}=\sum_{\mathrm{j}=1}^{\mathrm{v}} \phi_{2 \mathrm{j}} \dot{\mathrm{q}}_{\mathrm{j}} \quad, \quad \ddot{\mathrm{u}}_{2}=\sum_{\mathrm{j}=1}^{\mathrm{v}} \phi_{2 \mathrm{j}} \ddot{\mathrm{q}}_{\mathrm{j}}
$$

Recalling equation 4, the partial velocities of point $P$ in frame $\mathrm{N},{ }^{\mathrm{N}} \overrightarrow{\mathrm{v}}_{\mathrm{i}}^{\mathrm{P}}$, are given by

$\mathrm{N}_{\mathrm{v}_{\mathrm{i}}}^{\mathrm{P}}=\left[\phi_{1 \mathrm{i}}-\sum_{\mathrm{j}=1}^{\mathrm{v}}\left(\int_{0}^{\mathrm{x}} \phi_{2 \mathrm{i}}^{\prime} \phi_{2 \mathrm{j}}^{\prime} \mathrm{d} \sigma\right) \mathrm{q}_{\mathrm{j}}\right] \overrightarrow{\mathrm{a}}_{1}+\phi_{2 \mathrm{i}} \overrightarrow{\mathrm{a}}_{2}, \quad \mathrm{i}=1, \ldots, v$

The generalized inertia force is developed from equations 3,10 , and 20.

$$
\begin{aligned}
& F_{i}^{*}=-\int_{0}^{L} \rho\left\{\phi_{1 i}\left[\dot{v}_{1}+\ddot{u}_{1}-\dot{\omega}_{3} u_{2}-2 \omega_{3} \dot{u}_{2}-\omega_{3} v_{2}-\omega_{3}^{2}\left(x+u_{1}\right)\right]\right. \\
& -\left[\sum_{j=1}^{v}\left(\int_{0}^{x} \dot{\phi}_{2 j} \dot{\phi}_{2 k}^{\prime} d \sigma\right) q_{j}\right]\left[\dot{v}_{1}+\ddot{u}_{1}-\dot{\omega}_{3} u_{2}-2 \omega_{3} \dot{u}_{2}-\omega_{3} v_{2}\right. \\
& \left.-\omega_{3}^{2}\left(x+u_{1}\right)\right]+\phi_{2 i}\left[\dot{v}_{2}+\ddot{u}_{2}+\dot{\omega}_{3}\left(x+u_{1}\right)+2 \omega_{3} \dot{u}_{1}+\omega_{3} v_{1}\right. \\
& \left.\left.-\omega_{3}^{2} u_{2}\right]\right\} d x, \quad i=1,2, \ldots, v
\end{aligned}
$$

Substitution of the expressions in equations $12,15,16,17,18$, and 19 into equation 21 results in nonlinear terms in $\mathrm{q}_{j}, \dot{\mathrm{q}}_{j}$, and $\ddot{q}_{j}$. Because only small elastic deformations are of interest, the resulting expression for the generalized inertia force is linearized in $\mathrm{q}_{\mathrm{j}}$ and its time derivatives. Also, integration by parts is applied to eliminate the indefinite integral arising from the expressions for $\mathrm{u}_{1}, \dot{\mathrm{u}}_{1}$, and $\ddot{\mathrm{u}}_{1}$.

The final simplified form of the generalized inertia force is:

$$
\begin{aligned}
& -F_{i}^{*}=\left(\dot{v}_{1}-\omega_{3} v_{2}\right) \bar{W}_{1 i}+\left(\dot{v}_{2}+\omega_{3} v_{1}\right) \bar{W}_{2 i}-\omega_{3}^{2} \bar{X}_{1 i} \\
& +\dot{\omega}_{3} \bar{X}_{2 i}+\sum_{j=1}^{v} M 1_{i j} \ddot{q}_{j}+2 \omega_{3} \sum_{j=1}^{v} G 1_{i j} \dot{q}_{j}-\omega_{3}^{2} \sum_{j=1}^{v} M 1_{i j} q_{j} \\
& +\dot{\omega}_{3} \sum_{j=1}^{v} M 1_{i j} q_{j}-\left(\dot{v}_{1}-\omega_{3} v_{2}\right) \sum_{j=1}^{v} I P 1_{i j} q_{j} \\
& +\omega_{3}^{2} \sum_{j=1}^{v} I P 2_{i j} q_{j}, \quad i=1,2, \ldots, v
\end{aligned}
$$

where

$$
\begin{aligned}
& M 1_{i j}=W_{11 i j}+W_{22 i j}, \quad G 1_{i j}=W_{21 i j}-W_{12 i j} \\
& \bar{W}_{k i} \equiv \int_{0}^{L} \rho \phi_{k i} d x, \quad W_{k m i j} \equiv \int_{0}^{L} \rho \phi_{k i} \phi_{m j} d x \\
& \bar{X}_{k i} \equiv \int_{0}^{L} \rho x \phi_{k i} d x
\end{aligned}
$$

and terms resulting from the aforementioned integration by parts are: 


$$
\begin{aligned}
& I P 1_{i j}=\rho \int_{0}^{L}(L-x) \phi_{2 i}^{\prime} \phi_{2 j}^{\prime} d x \\
& I P 2_{i j}=\frac{1}{2} \rho \int_{0}^{L}\left(L^{2}-x^{2}\right) \phi_{2 i}^{\prime} \phi_{2 j}^{\prime} d x
\end{aligned}
$$

\section{Generalized_Active Force and System_Differential Equations}

Only axial and bending contributions to the strain energy function introduced in equation 6 will be considered. Hence,

$\mathrm{V}=\mathrm{V}_{\mathrm{axial}}+\mathrm{V}_{\text {bend }}$

Using Euler-Bernoulli beam theory, the bending strain energy can be expressed as:

$V_{\text {bend }}=\frac{1}{2} \int_{0}^{L} E I_{3} \frac{\partial^{2} u_{2}}{\partial x^{2}} d x$

where $\mathrm{E}$ is Young's Modulus, and $\mathrm{I}_{3}$ is the area moment of inertia of the beam about the $\vec{a}_{3}$ axis.

Assuming constant stress and strain across the cross section, the axial strain energy is

$V_{\text {axial }}=\frac{1}{2} E A_{b} \varepsilon_{x}^{2} d x$

where $A_{b}$ is the cross sectional area of the beam, and $\varepsilon_{x}$ is the axial strain given by

$\varepsilon_{x}=\frac{\partial s}{\partial x}$

Using equations $30-33$, the total strain energy is

$V=\frac{1}{2} \int_{0}^{L} E A_{b}\left(\frac{\partial s}{\partial x}\right)^{2} d x+\frac{1}{2} \int_{0}^{L} E I_{3} \frac{\partial^{2} u_{2}}{\partial x^{2}} d x$

After appropriate substintion, partial differentiation, and rearranging, the final form of the generalized active force is:

$$
\begin{aligned}
& -F_{i}=\sum_{j=1}^{v}\left[\int_{0}^{L} E A_{b} \phi_{1 i}^{\prime} \phi_{1 j}^{\prime} d x\right] q_{j} \\
& +\sum_{j=1}^{v}\left[\int_{0}^{L} E_{3} \phi_{2 i}^{\prime \prime} \phi_{2 j}^{\prime \prime} d x\right] q_{j}, \quad i=1,2, \ldots, v
\end{aligned}
$$

Using Kane's dynamical equations (equation 2), the resulting system differential equations are:

$$
\begin{aligned}
& \sum_{j=1}^{v} M 1_{i j} \ddot{q}_{j}+2 \omega_{3} \sum_{j=1}^{v} G 1_{i j} \dot{q}_{j}-\omega_{3}^{2} \sum_{j=1}^{v} M 1_{i j} q_{j} \\
& +\dot{\omega}_{3} \sum_{j=1}^{v} G 1_{i j} q_{j}-\left(\dot{v}_{1}-\omega_{3} v_{2}\right) \sum_{j=1}^{v} I P 1_{i j} q_{j}+\omega_{3}^{2} \sum_{j=1}^{v} I P 2_{i j} q_{j}
\end{aligned}
$$

$+\sum_{j=1}^{v} K 1_{i j} q_{j}=-\left(\dot{v}_{1}-\omega_{3} v_{2}\right) \bar{W}_{1 i}-\left(\dot{v}_{2}+\omega_{3} v_{1}\right) \bar{W}_{2 i}+\omega_{3}^{2} \bar{X}_{1 i}$

$-\dot{\omega}_{3} \bar{X}_{2 \mathrm{i}}, \quad \mathrm{i}=1,2, \ldots, \mathrm{V}$

where

$K 1_{i j}=\int_{0}^{L} E A_{b} \phi_{1 i}^{\prime} \phi_{1 j}^{\prime} d x+\int_{0}^{L} E I_{3} \phi_{2 i}^{\prime \prime} \phi_{2 j}^{\prime \prime} d x$

In the AIGC approach a set of algebraic constraints are added to the system differential equations to form the equations of motion. The algebraic constraints are introduced to overcome the failure of the IGC approach to insure satisfaction of the boundary conditions for membrane-dominated problems. Additional insight into the utility of the algebraic constraints can be gained by investigating the failure of the NSD approach to insure satisfaction of the boundary conditions for bendingdominated problems.

\section{Enforcement of Boundary Conditions}

For the bending-stiffness-dominated beam problem depicted in Figure 2, the shape functions are obtained from solving axial and bending vibration problems with the respective boundary conditions

$$
\begin{array}{lll}
\left.\phi_{1 \mathrm{j}}\right|_{\mathrm{x}=0}=0 & , & \left.\frac{\partial \phi_{1 \mathrm{j}}}{\partial \mathrm{x}}\right|_{\mathrm{x}=\mathrm{L}}=0 \\
\left.\phi_{2 \mathrm{j}}\right|_{\mathrm{x}=0}=0 & , & \left.\frac{\partial^{2} \phi_{2 \mathrm{j}}}{\partial \mathrm{x}^{2}}\right|_{\mathrm{x}=\mathrm{L}}=0 \\
\left.\frac{\partial \phi_{2 \mathrm{j}}}{\partial \mathrm{x}}\right|_{\mathrm{x}=0}=0 & , & \left.\frac{\partial^{3} \phi_{2 \mathrm{j}}}{\partial \mathrm{x}^{3}}\right|_{x=L}=0
\end{array}
$$

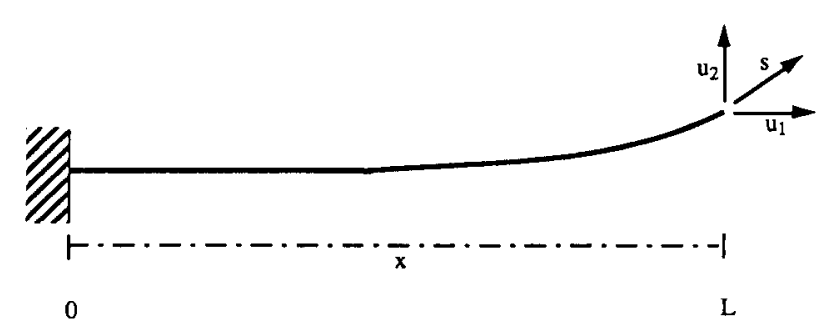

Boundary Conditions
$\left.u_{1}\right|_{x=0}=0$, or $\left.s\right|_{x=0}=0$
$\left.\frac{\partial s}{\partial x}\right|_{x=L}=0$
$\left.\mathrm{u}_{2}\right|_{x=0}=0$
$\left.\frac{\partial^{2} U_{2}}{\partial x^{2}}\right|_{x=L}=0$
$\left.\frac{\partial u_{2}}{\partial x}\right|_{x=0}=0$
$\left.\frac{\partial^{3} u_{2}}{\partial x^{3}}\right|_{x=L}=0$

Figure 2 -- Boundary Conditions for a Cantilever Beam 
Using these these choices for the shape functions in equations 11 and 12 (IGC approach), it is seen that the boundary conditions set forth in Figure 2 are fully satisfied. On the other hand, in the initial NSD approach, the deformation measures were taken to be

$\mathrm{u}_{1}=\sum_{\mathrm{j}=1}^{\mathrm{v}} \phi_{1 \mathrm{j}}(\mathrm{x}) \mathrm{q}_{\mathrm{j}}(\mathrm{t}) \quad, \quad \mathrm{u}_{2}=\sum_{\mathrm{j}=1}^{\mathrm{v}} \phi_{2 \mathrm{j}}(\mathrm{x}) \mathrm{q}_{\mathrm{j}}(\mathrm{t})$

with $\phi_{1 \mathrm{j}}$ and $\phi_{2 \mathrm{j}}$ as before. Hence, in this representation,

instead of enforcing $\left.\frac{\partial s}{\partial x}\right|_{x=L}=0$, the condition $\left.\frac{\partial u_{1}}{\partial x}\right|_{x=L}=0$ is enforced. Yoo recognized this deficiency and attempted to remedy it through the use of so-called interaction modes (between $u_{1}$ and $u_{2}$ ) generated by enforcing an inextensibility condition. Some improvement was found, but it did not work for some circumstances (as will be shown shortly).

For the membrane-stiffness-dominated beam problem shown in Figure 3 , the shape functions are obtained from vibration analyses in which the following boundary conditions are enforced.

$$
\begin{array}{lll}
\left.\phi_{1 j}\right|_{x=0}=0 & , & \left.\phi_{1 j}\right|_{x=L}=0 \\
\left.\phi_{2 j}\right|_{x=0}=0 & \left.\phi_{2 j}\right|_{x=L}=0 \\
\left.\frac{\partial^{2} \phi_{2 j}}{\partial x^{2}}\right|_{x=0}=0 & \left.\frac{\partial^{2} \phi_{2 j}}{\partial x^{2}}\right|_{x=L}=0
\end{array}
$$

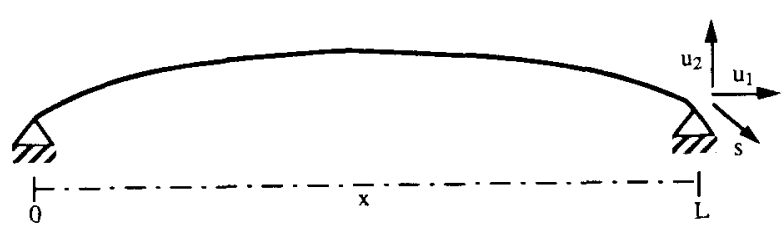

Boundary Conditions

$$
\begin{array}{ll}
\left.u_{1}\right|_{x=0}=0, \text { or }\left.s\right|_{x=0}=0 & \left.u_{1}\right|_{x=L}=0 \\
\left.u_{2}\right|_{x=0}=0 & \left.u_{2}\right|_{x=L}=0 \\
\left.\frac{\partial^{2} u_{2}}{\partial x^{2}}\right|_{x=0}=0 & \left.\frac{\partial^{2} u_{2}}{\partial x^{2}}\right|_{x=L}=0
\end{array}
$$

Figure 3 -- Boundary Conditions for a Pinned-Pinned Beam

Thus the NSD approach, using these shape functions in equations 44 and 45 , fully satisfies the boundary conditions shown in Figure 3. Note that in the IGC approach the condition $\left.\mathrm{s}\right|_{\mathrm{x}=\mathrm{L}}=0$ is enforced instead of $\left.\mathrm{u}_{1}\right|_{\mathrm{x}=\mathrm{L}}=0$.

By using the shape function summation approach, only boundary conditions explicit in the chosen deformation measures can be enforced. Thus only boundary conditions expressed in $\mathrm{s}$ and $\mathrm{u}_{2}$ can be enforced for the IGC approach and only boundary conditions expressed in $u_{1}$ and $u_{2}$ can be enforced for the NSD approach. This prevents either approach from accurately addressing both the bending and membrane problems, without some modification.

Boundary conditions which are not explicit in the chosen deformation measures can be enforced by adding constraints derived from evaluation of equation 15 , which represents the the geometric relationship between $u_{1}, s$, and $u_{2}$. Recall that in the IGC approach, the condition that $u_{1}$ be zero at the right end of the beam (membrane-dominated problem) could not be satisfied. Using equation 15 this boundary condition becomes the following constraint between the generalized coordinates:

$0=\sum_{j=1}^{v} \phi_{1 j}(L) q_{j}-\frac{1}{2} \sum_{j=1}^{v} \sum_{k=1}^{v}\left[\int_{0}^{L} \phi_{2 j}^{\prime} \phi_{2 k}^{\prime} d x\right] q_{j} q_{k}$

This is an example of the general approach for enforcing boundary conditions. However, care must be taken in selecting the shape functions so that undesirable conditions do not arise. For example, using the shape functions described above in the IGC approach, the constraint (equation 52) could, possibly, be satisfied. However, zero stretch at the right end of the beam would also result which is not physically correct. Clearly what is needed is an approach which allows arbitrary boundary conditions to be prescribed. This is achieved by using an approach similar to one set forth by Craig and Bampton in work on dynamic system substructuring. This will now be described.

\section{Shape Functions. Constraints, and Complete Equations of Motion}

Craig and Bampton use dynamic modes in combination with static displacement modes in a substructuring approach for vibration problems. The dynamic modes, referred to as fixedinterface modes, are developed from an eigenanalysis of the lateral vibrations of a beam with boundary conditions of no slope and displacement at the ends of the beam. The static modes, referred to as constraint modes, are obtained by applying unit displacements or rotations in the directions held fixed in developing the dynamic modes. While enforcing each unit displacement or rotation, the others are held fixed. For example, one such shape function satisfies the conditions

$\phi(0)=1$, and $\phi^{\prime}(0)=\phi(L)=\phi^{\prime}(L)=0$. The combined set of shape functions, static and dynamic, is capable of representing any arbitrary boundary condition. The approach for developing the modes to describe the $s$ and $u_{2}$ deformations is slightly different and are outlined below.

The dynamic shape functions for the $u_{2}$ deformation are the eigenfunctions obtained from a linear lateral vibration analysis of a beam in a non-rotating frame with no displacement or slope at either end. The static shape functions are polynomials developed to satisfy the enforced displacement or slope conditions. Third degree polynomials are used because four boundary conditions need to be satisfied. Figure 4 shows the static shape functions and the polynomials that represent them. The use of polynomials for the static modes is a deviation from the approach of Craig and Bampton. To follow their approach completely, each unit deflection condition would have to be solved using beam theory. As the focus here is to demonstrate that a formulation can be developed that improves upon the predictive capabilities of the IGC and NSD approaches by ensuring that the boundary conditions are met, use of the polynomials, which yield the necessary boundary condition flexibility is deemed to be sufficient. 


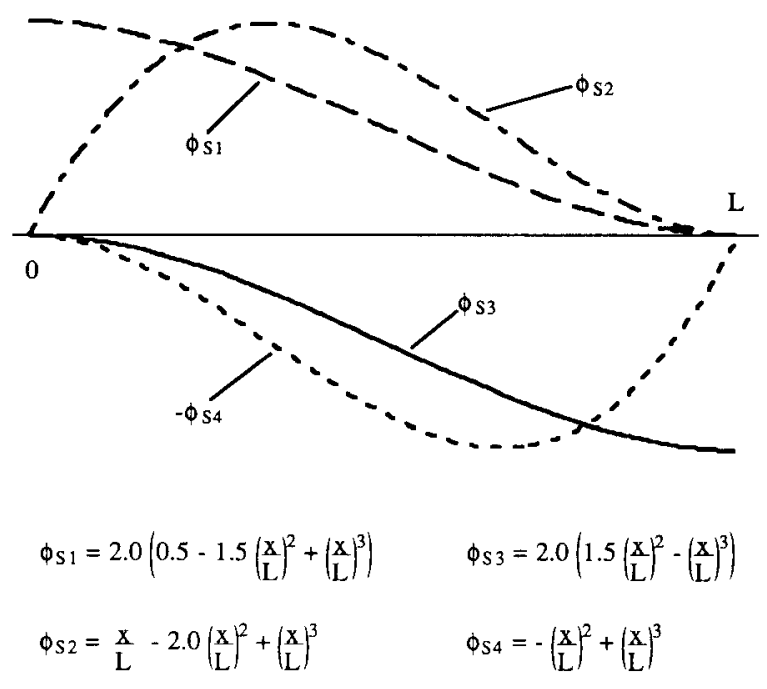

Figure 4 -- Static Modes for Lateral Deformation Description

The development of the shape functions to describe the $\mathrm{s}$ deformation is as follows. The dynamic shape functions are derived from an eigenanalysis of the axial vibrations of a rod with no displacement at either end. The supplementary static modes are developed from polynomials which satisfy unit displacements at one end and no displacement at the other end.

The constraint equations for the bending and membrane problems are now set forth.

\section{* Bending Constraints}

$$
\begin{array}{lll}
\sum_{j=1}^{v} \phi_{1 j}(0) q_{j}=0 & , & \sum_{j=1}^{v} \phi_{2 j}(0) q_{j}=0 \\
\sum_{j=1}^{v} \phi_{2 j}^{\prime}(0) q_{j}=0 & , & \sum_{j=1}^{v} \phi_{1 j}^{\prime}(L) q_{j}=0 \\
\sum_{j=1}^{v} \phi_{2 j}^{\prime \prime \prime}(L) q_{j}=0 & , & \sum_{j=1}^{v} \phi_{2 j}^{\prime \prime}(L) q_{j}=0
\end{array}
$$

\section{* Membrane Constraints}

$$
\begin{aligned}
& \sum_{j=1}^{v} \phi_{1 j}(0) q_{j}=0 \quad, \quad \sum_{j=1}^{v} \phi_{2 j}(0) q_{j}=0 \\
& \sum_{j=1}^{v} \phi_{2 j}^{*}(0) q_{j}=0 \\
& \sum_{j=1}^{v} \phi_{1 j}(L) q_{j}-\frac{1}{2} \sum_{j=1}^{v} \sum_{k=1}^{v}\left[\int_{0}^{L} \phi_{2 j}^{\prime} \phi_{2 k}^{\prime} d x\right] q_{j} q_{k}=0 \\
& \sum_{j=1}^{v} \phi_{2 j}(L) q_{j}=0 \quad, \quad \sum_{j=1}^{v} \phi_{2 j}^{\prime r}(L) q_{j}=0
\end{aligned}
$$

Thus the complete differential algebraic equations of motion for the membrane problem using the AIGC approach are the ODEs in equation 36, along with the constraints in equations 53a-53f. For the membrane problem, the ODEs in equation 36 in addition to the constraints in equations $54 \mathrm{a}-54 \mathrm{f}$ describe the system.

Despite the fact that the AIGC approach provides different constraints for the bending and membrane problems (equations 53 and 54, respectively), the approach is problem independent. This is true because the constraints are handled numerically in a generic way, and therefore differences in the specific constraints do not affect the solution procedure. That different boundary conditions must be specified for each given problem, is simply part of the problem description, and is necessary in any approach.

Here, numerical solutions to the differential algebraic equations of motion are obtained using the approach developed by Baungarte 9

\section{Verification Problems, Solutions, and Interpretation}

The failure of the IGC and NSD approaches to solve both bending and membrane dominated problems, and the ability of the AIGC approach to solve both problems will be demonstrated by investigating two verification problems.

A bending-stiffness-dominated problem, studied in reference [3], is shown in Figure 5. It involves a flexible beam cantilevered to a rotating base. The angular velocity of the beam base, which is a prescribed function of time, is given by:

$\omega(t)= \begin{cases}\omega_{s}\left[6\left(\frac{t}{T_{s}}\right)^{5}-15\left(\frac{t}{T_{s}}\right)^{4}+10\left(\frac{t}{T_{s}}\right)^{3}\right] & \text {, if } 0 \leq t \leq T_{s} \\ \omega_{s} & , \text { if } t>T_{s}\end{cases}$

where: $\quad \omega_{\mathrm{s}}=\frac{\pi}{10} \mathrm{rad} / \mathrm{sec}, \mathrm{T}_{\mathrm{s}}=1 \mathrm{sec}$

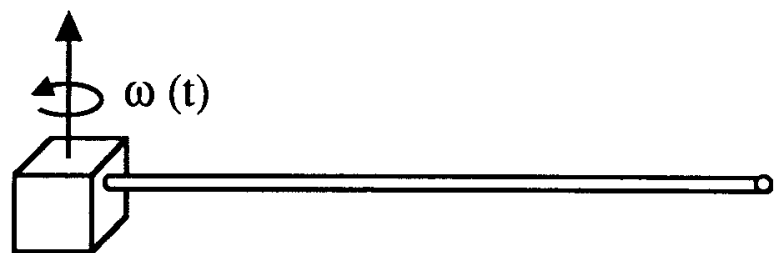

$\begin{array}{ll}\text { Mass per Unit Length } & \rho=250 \mathrm{~kg} / \mathrm{m} \\ \text { Young's Modulus } & \mathrm{E}=6.89 \times 10^{9} \mathrm{~N} / \mathrm{m}^{2} \\ \text { Length } & \mathrm{L}=30.5 \mathrm{~m} \\ \text { Cross Sectional Area } & \mathrm{A}_{\mathrm{b}}=9.30 \times 10^{-2} \mathrm{~m}^{2} \\ \text { Area Moment of Inertia } & \mathrm{I}_{3}=7.20 \times 10^{-4} \mathrm{~m}^{4}\end{array}$

Figure 5 -- Bending-Dominated Validation Problem

The lateral deflection response of the free end of the beam given in reference [3] is shown in Figure 6a. The responses for the IGC and NSD approaches (reference [6]) are given in Figure 
$6 \mathrm{~b}$, and the response for the AIGC approach is shown in Figure $6 \mathrm{c}$. Both the IGC and AIGC solutions agree with the results in reference [3]. However, it is seen that the NSD (even with the inclusion of Yoo's "interaction modes") method fails to correctly solve this problem.

Lateral Tip Deflection vs. Time

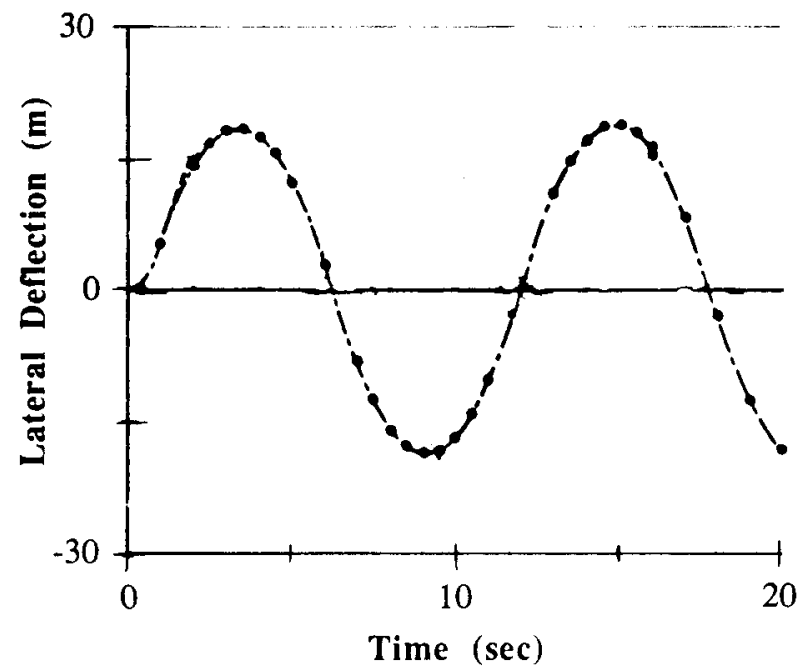

Figure 6a -- Christensen and Lee's Results for the BendingDominated Problem

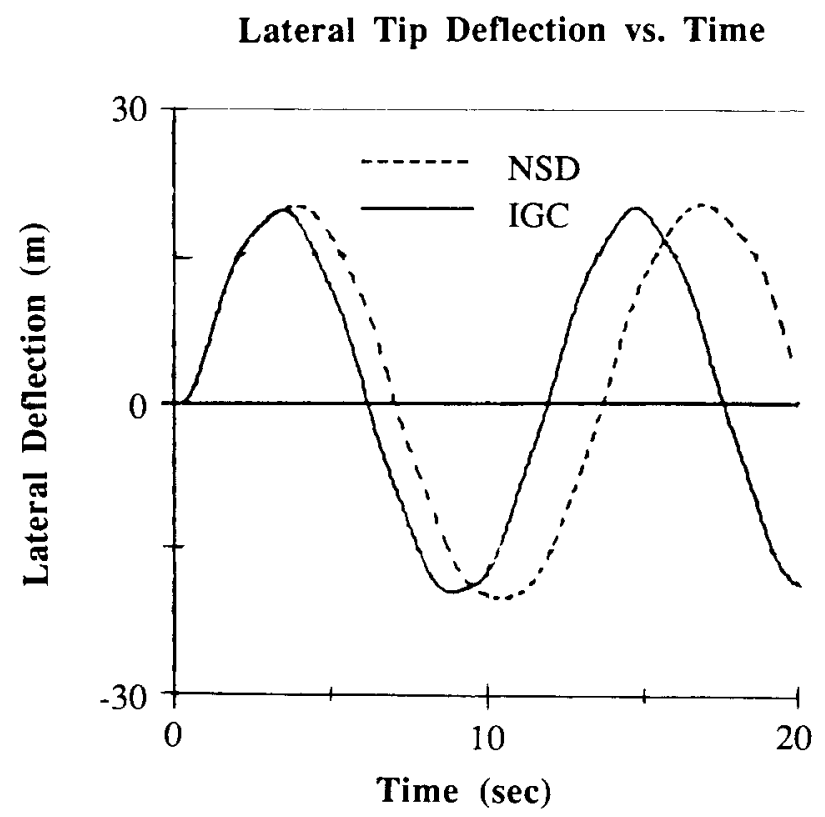

Figure $6 \mathrm{~b}$-- IGC and NSD Results for the Bending-Dominated Problem
Lateral Tip Deflection vs. Time

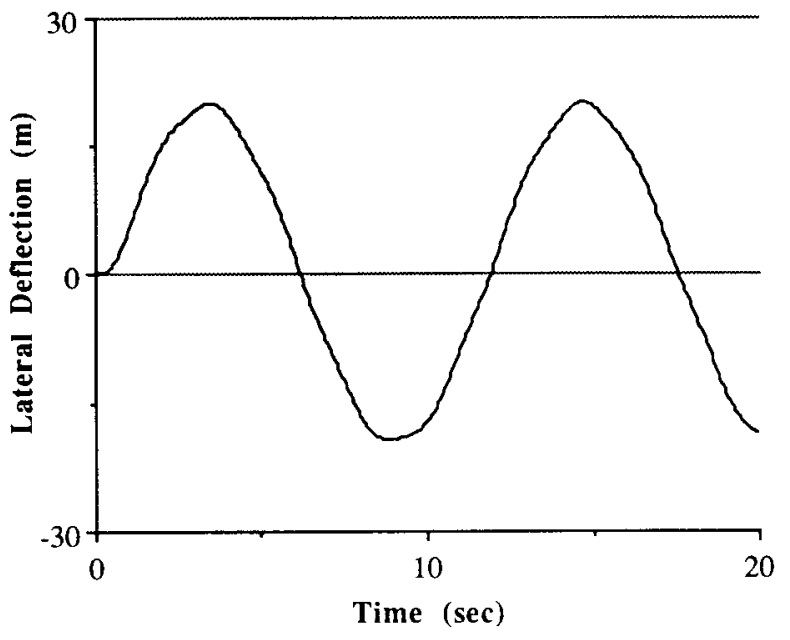

Figure $6 c$-- AIGC Results for the Bending-Dominated Problem

A membrane-stiffness-dominated problem, studied in reference [6] is shown in Figure 7. It is comprised of a flexible beam pinned at the center and outer edge of a rigid rotating table. The prescribed angular velocity of the table is given by:

$\omega(t)= \begin{cases}\frac{\omega_{s}}{T_{s}}\left[t-\left(\frac{T_{s}}{2 \pi}\right) \sin \left(\frac{2 \pi t}{T_{s}}\right)\right] & \text { if } 0 \leq t \leq T_{s} \\ \omega_{s} & \text { if } t>T_{s}\end{cases}$

where: $\omega_{\mathrm{s}}=6 \mathrm{rad} / \mathrm{sec}, T_{\mathrm{s}}=15 \mathrm{sec}$

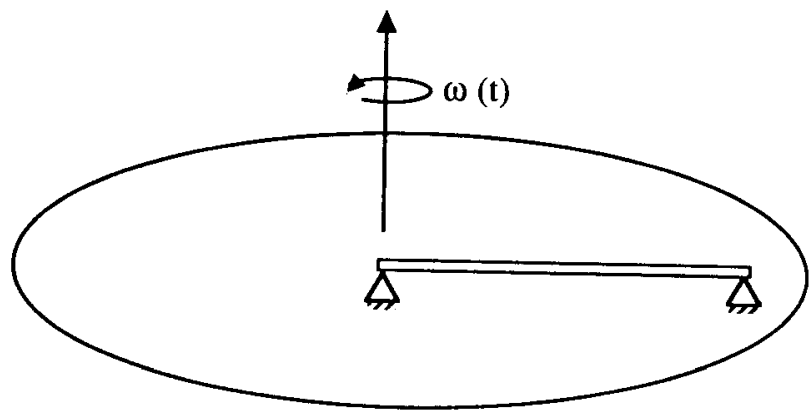

$\begin{array}{ll}\text { Mass per Unit Length } & \rho=1.2 \mathrm{~kg} / \mathrm{m} \\ \text { Young's Modulus } & E=7.0 \times 10^{10} \mathrm{~N} / \mathrm{m}^{2} \\ \text { Length } & \mathrm{L}=20 \mathrm{~m} \\ \text { Cross Sectional Area } & \mathrm{A}_{b}=4.0 \times 10^{-4} \mathrm{~m}^{2} \\ \text { Area Moment of Inertia } & \mathrm{I}_{3}=2.0 \times 10^{-7} \mathrm{~m}^{4}\end{array}$

Figure 7 -- Membrane-Dominated Validation Problem

The solutions predicted by the AIGC, IGC and NSD approaches, for the lateral deflection of a point at the beam midspan, are shown in Figure 8 The solutions predicted by the NSD and AIGC approaches are nearly identical, and are not 
individually identifiable. Yoo verified that the solution predicted by the NSD approach, and hence also by the AIGC approach, is correct through the use of an independent calculation of maximum deflection, based on a static structural analogy, and an ADAMS ${ }^{10}$ transient solution. Thus, the solutions shown in Figure 8 demonstrate the ability of the NSD and AIGC approaches, and the inability of the IGC approach, to solve the membrane-stiffness-dominated problem.

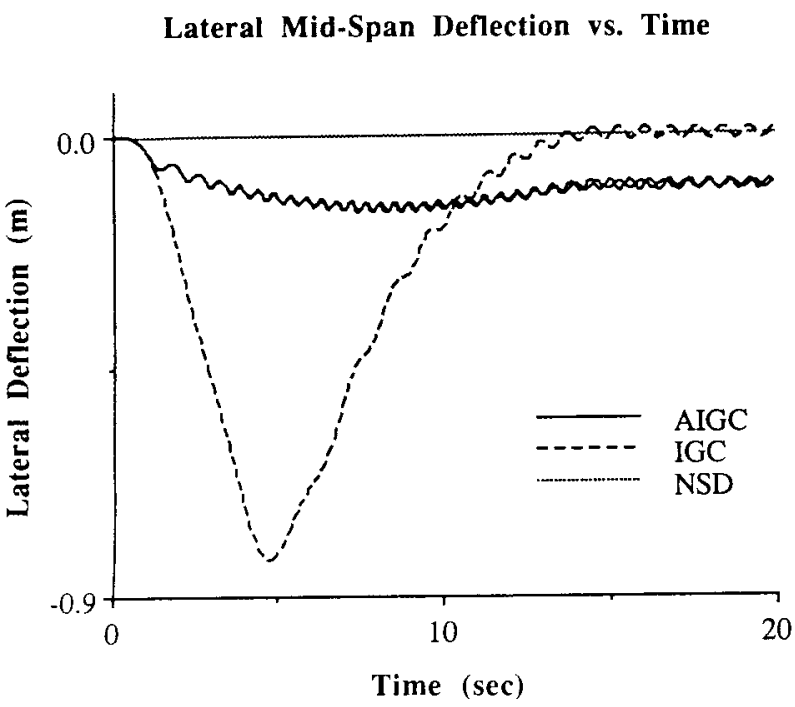

Figure 8 -- AIGC, IGC and NSD Results for the MembraneDominated Problem

An interesting feature of the results in Figure 8 is that the steady state non-zero lateral deformation predicted by the NSD and AIGC approaches indicates that a buckling-type behavior occurs. Note that this is not predicted by the IGC formulation. The existence of such behavior was verified by a NASTRAN 11 linear buckling analysis which predicted a first critical angular velocity $\left(\omega_{\mathrm{s}}\right)$ of $3.01 \mathrm{rad} / \mathrm{sec}$. As seen in Figure $9\left(\omega_{\mathrm{s}}=1.0\right.$ $\mathrm{rad} / \mathrm{sec})$, at values of $\omega_{\mathrm{s}}$ below the critical speed, no bucklingtype behavior is exhibited.

\section{Extension to Qther Physical Problems}

While only problems with cantilevered or pinned-pinned beam attachment have been addressed in this work, problems with other physical attachment could be analyzed with the AIGC approach. This would be done by using the boundary conditions for the specific problem in question. After describing the boundary conditions mathematically in terms of $s, u_{1}$, and $u_{2}$, the appropriate algebraic constraints could be obtained. As is done in this work, the complete equations of motion would then be obtained by addition of the set of second order differential equations (equation 36).

The development of the AIGC approach is limited here to twodimensional prescribed motion of a single beam. Currently efforts are underway to extend the formulation to permit threedimensional motion and deformation as well as applied forces/torques.
Lateral Mid-Span Deflection vs. Time

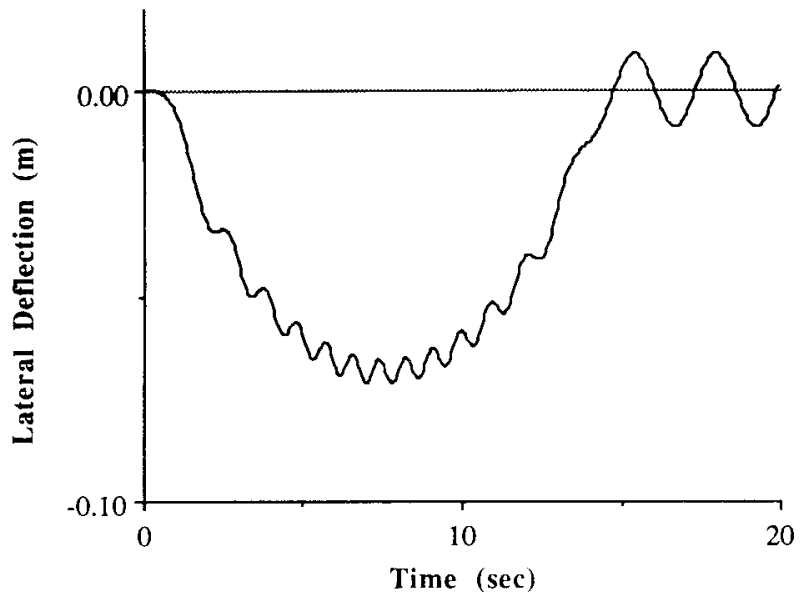

Figure 9 -- Membrane-Stiffness-Dominated Problem Solution at a Sub-Critical Speed

\section{Summary}

A new flexible body dynamic beam formulation, the Augmented Imbedded Geometric approach, has been developed. The ability of this approach to improve upon two existing approaches, the Imbedded Geometric Constraint and Nonlinear Strain Displacement approaches, has been demonstrated through the use of two specific problems. Application of this approach to problems with other boundary conditions has been discussed.

\section{References}

1 Simo, J. C., Vu Quoc, L., "A Finite Strain Beam Formulation. The Three-Dimensional Dynamics Problem. Part I.," Computer Methods in Applied Mechanics and Engineering, Vol. 49, 1985, pp. 55-70.

2 Simo, J. C., Vu Quoc, L., "On the Dynamics of Flexible Beams Under Large Overall Motions - The Plane Case: Parts I \& II," Journal of Applied Mechanics,Dec. 1986, Vol. 53, pp. 849-863.

3 Christensen, E. R., and Lee, S. W., "Nonlinear Finite Element Modeling of the Dynamics of Unrestrained Flexible Structures," Computers and Structures, Vol. 23, 1986, pp. 819-829.

4 Kane, T. R., Ryan, R. R., and Bannerjee A. K., "Dynamics of a Cantilever Beam Attached to a Moving Base," Journal of Guidance, Control, and Dynamics, Vol. 10, March-April, 1987, pp. 139-151.

5 Ryan, R. R., "Simulation of Actively-Controled Flexible Spacecraft," Journal of Guidance, Control, and Dynamics, Vol. 13, July-August, 1990, pp. 691-702.

6 Yoo, H. H., "Dynamic Modeling of Flexible Bodies in Multibody Systems," Ph.D. Thesis, The University of Michigan, 1989.

7 Kane, T. R., and Levinson, D. A., Dynamics, Theory and Applications, McGraw-Hill, New York, N.Y., 1983 
8 Craig, R. R., and Bampton, M. C. C., "Coupling of Substructures for Dynamic Analysis," AIAA Journal, Vol 3, 1968, pp. 1313-1319.

9 Baumgarte, J. W., "Stabilization of Constraints and Integrals of Motion in dynamical Systems," Computational Methods in Applied Mechanics and Engineering, Vol. 1, 1972, pp. 1-16.

10 ADAMS 5.2 User's Manual, Mechanical Dynamics Inc., Ann Arbor, MI, April 1987.

11 MSC/NASTRAN User's Manual Version 66A, The MacNeal-Schwendler Corporation, Los Angles, CA, November 1989. 